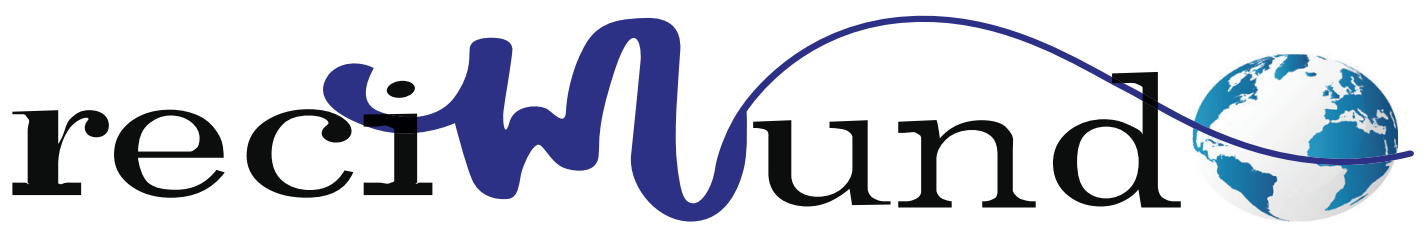

Revista Científica Mundo de la Investigación y el Conocimiento

DOI: 10.26820/recimundo/4.(1).enero.2020.173-182

URL: http://recimundo.com/index.php/es/article/view/755

EDITORIAL: Saberes del Conocimiento

REVISTA: RECIMUNDO

ISSN: 2588-073X

TIPO DE INVESTIGACióN: Artículo de Revisión

CóDIGO UNESCO: 3304 Tecnología de Los Ordenadores;

5311 Organización y Dirección de Empresas

PAGINAS: $173-182$

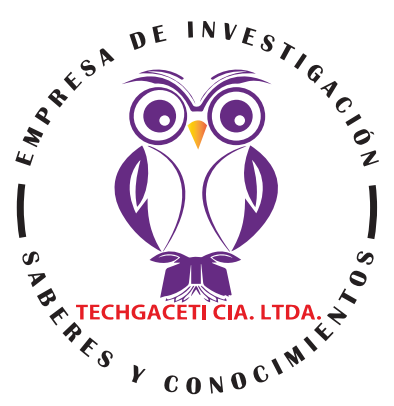

\title{
El impacto de las redes sociales en la administración de empresas
}

The impact of social networks in business administration

\section{0 impacto das redes sociais na administração de empresas}

Francisco Xavier Hugo Cardenas1; César Eduardo Jimenez Rosero2; Mykola Holovatyi3; Paul Eduardo Lara Pazos ${ }^{4}$

RECIBIDO: 18/09/2019 ACEPTADO: 29/10/2019 PUBLICADO: 31/01/2020

1. Magister en Seguridad Salud y Ambiente; Ingeniero Ambiental; Investigador Independiente; Guayaquil, Ecuador; fcohugo@hotmail.com; (D) https://orcid.org/0000-0002-8102-8931

2. Magister en Seguridad y Salud Ocupacional; Ingeniero en Maderas; Investigador Independiente; Guayaquil, Ecuador; cesarjimenezrosero@outlook.com; (D) https://orcid.org/0000-0002-1085-3774

3. Investigador Independiente; Guayaquil, Ecuador, Ecuador; m.f.holovaty@ukr.net; (D) https://orcid.org/00000002-5838-417X

4. Máster en Project Management; Magister en Gestión de la Industria de los Hidrocarburos; Ingeniero de Petróleos; Investigador Independiente; Guayaquil, Ecuador; edu65lara@yahoo.es; (D) https://orcid.org/0000-00026451-0179

CORRESPONDENCIA

Francisco Xavier Hugo Cardenas

fcohugo@hotmail.com

Guayaquil, Ecuador

○ RECIMUNDO; Editorial Saberes del Conocimiento, 2020 


\section{RESUMEN}

Las Redes Sociales Virtuales son una rama de aplicación de las tecnologías de la información y las comunicaciones que actualmente juegan un rol fundamental en la sociedad. En este sentido, la actividad económica, las empresas, organizaciones y demás actividades productivas se han adaptado a nuevos modelos de gestión donde las tecnologías de información y comunicación tengan el protagonismo necesario.

En efecto, la administración de las empresas hoy en día usa las interfaces entre redes sociales como principal herramienta de gestión.

Con la elaboración del presente trabajo, se pretende exponer los diversos conceptos asociados a las redes sociales, su historia y tipología. La relación que guarda la administración con el uso de las tecnologías de la información y las redes. Se hará especial énfasis en el impacto proporcionado por las mismas en el ámbito de la administración de empresas. Más que afirmaciones se esbozarán conjunto o a nivel individual.

Palabras clave: Red Social, Tecnología de la información, Web 2.0, Administración, Empresas, Redes, media, comunicaciones, internet en las empresas.

\section{ABSTRACT}

Virtual Social Networks are a branch of application of information and communications technologies that currently play a fundamental role in society. In this sense, economic activity, companies, organizations and other productive activities have adapted to new management models where information and communication technologies have the necessary role.

Indeed, business administration today uses interfaces between social networks as the main management tool.

With the preparation of this work, it is intended to expose the various concepts associated with social networks, their history and typology. The relationship between the administration and the use of information technologies and networks. Special emphasis will be placed on the impact provided by them in the field of business administration. More than affirmations will be outlined jointly or individually.

Keywords: Social Network, Information Technology, Web 2.0, Administration, Companies, Networks, media, communications, internet in companies.

\section{RESUMO}

As redes sociais virtuais são um ramo da aplicação das tecnologias da informação e comunicação que atualmente desempenham um papel fundamental na sociedade. Nesse sentido, a atividade econômica, empresas, organizações e outras atividades produtivas se adaptaram a novos modelos de gestão, onde as tecnologias da informação e comunicação têm o papel necessário.

De fato, a administração de empresas hoje usa interfaces entre redes sociais como a principal ferramenta de gerenciamento.

Com a elaboração deste trabalho, pretende-se expor os vários conceitos associados às redes sociais, sua história e tipologia. A relação entre administração e uso de tecnologias e redes de informação. Ênfase especial será dada ao impacto fornecido por eles no campo da administração de empresas. Mais do que afirmações serão descritas em conjunto ou individualmente.

Palavras-chave: Rede social, Tecnologia da informação, Web 2.0, Administração, Empresas, Redes, mídia, comunicação, internet nas empresas. 


\section{Introducción}

Indiscutiblemente el uso del Internet y las tecnologías de información han ganadoprotagonismo en todos los ámbitos de la vida diaria de las personas, en este sentido, en los últimos tiempos las grandes corporaciones poseedoras de las principales plataformas de redes sociales han salido al ruedo aprovechando este nuevo estilo de vida y tendencia, siendo utilizadas diariamente por miles de millones de personas.

El ámbitoorganizacional no escapade este fenómeno y es que, cada vez más empresas se unen al usointensivo de las tecnologías de la información y comunicaciones, hecho que les otorga la posibilidad de optimizar sus procesos productivos generando altos niveles de eficiencia con el uso de las mismas. Interfaces como las redes sociales,brindan a las organizaciones la posibilidad de ampliar y replantearmuchos de sus procesos habituales,tales como:lacomercialización, marketing o venta de productos y servicios, a fin de garantizar a los consumidores una óptima calidad de servicio.

Las redes sociales ofrecen una vía alterna a las empresas para llegar a los consumidores, estos sitios son el lugar más prominente donde estos últimos intercambian opiniones e ideas acerca de los productos y servicios de las compañías y donde éstas no tienen capacidad alguna para controlar esta información. Este hecho hace más atractiva todavía su adopción por las empresas ya que les permite conocer más profundamente a los consumidores a través de investigaciones de mercado y les posibilita también tener una mayor capacidad de respuesta ante múltiples situaciones. En este sentido, ¿se pudiera afirmar que las redes sociales no son una simple herramienta para las empresas, sino que se han convertido en una necesidad para las mismas?

Por otra parte, existen otras vertientes que plantean que el uso de las redes sociales en una organización puede convertirse en una potencial amenaza para la misma, ya quela productividad y el rendimiento de suscolaboradores pudiera verse comprometidopor la utilización excesiva de las mismas.

De tal manera, se plantea la interrogante ¿pueden las redes sociales ser beneficiosas para para la organización? o por el contrario ¿puede verse comprometida la productividad de la misma?

En el presente artículo se dilucidarán diversos escenarios ante la actual disyuntiva que enmarca la utilización de las redes sociales en la administración de las empresas, analizando la temática desde el punto de vista interno, en la interrelación que pueda tener la empresa con sus empleados, así como también desde el punto de vista externo y la relación que pueda tener la organización tanto con clientes como proveedores y otros relacionados.

\section{Metodología}

La metodología utilizada se basa en la investigación documental, a través de la revisión bibliográfica, de documentos de carácter científico, artículos, publicaciones académicas, tendencias en el mercado, medios electrónicos, de diversas índoles,; se constata las ideas comúnmente adoptadas por los especialistas, para en un análisis descriptivo exponer el principal impacto que tienen las redes en las organizaciones empresariales y en sus modelos de administración.

La utilización de las redes sociales se ha traducido en cambios significativos dentro del mundo empresarial, en consecuencia el objetivo de este trabajo, que es analizar el impacto que tiene para la administración de las empresas el uso de las Tecnologías de la Información y las Comunicaciones (TIC), del Internet y de las redes sociales. Se realizó una investigación de la bibliografía disponible, respecto al desarrollo tecnológico desde sus inicios hasta la actualidad. Se analizó la importancia, y diversos utilidades que tiene para las empresas o corporaciones el uso de estas tecnologías para su desarrollo

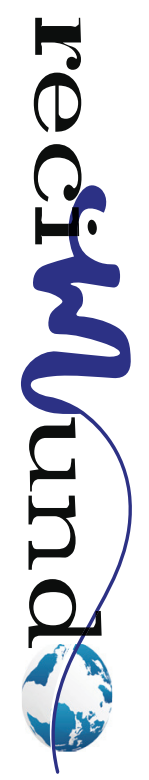


económico y para el logro de sus objetivos planteados, basándose en los beneficios para con los clientes, desarrollo interno de la empresa, captación y reclutamiento de personal, posicionamiento de marca, elaboración de estudios de mercado, marketing, publicidad, y comercialización.

\section{El impacto de las redes sociales en la ad- ministración de empresas}

El entorno versátil y pujante de los requerimientos de la sociedad actual dictamina el curso de las empresas, obligándolas a adaptarse replanteándose constantemente todos sus procesos, desde el inicio hasta el final de la cadena productiva.

En efecto, las organizaciones presentan su atención y dirigen sus recursos en función de optimizar:

- La percepción sus productos y servicios, a través del diseño y la generación de prototipos digitales.

- La fabricación, operación y se prestación de servicios;

- Construcción de las plataformas tecnológicas, para prestar la operación y el mantenimiento de manera satisfactoria.

En atención al inminente cambio social apalancado por las novedosas y atrayentes vías de comunicación como lo es el Internet, las organizaciones se encuentran en una constante adaptaciónbasándose en la conducta de los consumidores, tratandoinsistentemente de estar presentes donde ellos lo están y pasan su tiempo libre, es decir, en las redes sociales.En este sentido, cada vez más empresas deciden sacar provecho a ese tiempo en las redes y deciden adoptar estas interfaces.

\section{Administración de empresas}

Todas las actividades de producción de bienes y servicios son administradas por organizaciones, compuestas por personas y recursos físicos, materiales, financieros y tecnológicos, y éstas guardan una interrela- ción de mutua dependencia.

"En la sociedad moderna, las personas nacen, crecen, aprenden, se relacionan y mueren dentro de organizaciones. Éstas son extremadamente heterogéneas y diversas, cuyo tamaño, características, estructuras y objetivos son diferentes. Existen organizaciones lucrativas, llamadas empresas. La administración no es otra cosa que la dirección racional de las actividades de una organización, Ella implica planeación, organización, dirección y control de las actividades realizadas en una empresa, diferenciadas por la división del trabajo. Por tanto, la administración es imprescindible para la existencia, la supervivencia y el éxito de las organizaciones. Sin la administración, las organizaciones jamás tendrían condiciones que les permitan existir y crecer. La Teoría General de la Administración es el campo del conocimiento humano que se ocupa del estudio de la Administración en general, sin importar dónde se aplique." (Chiavenato, 2007)

Se hacen indispensables para el adecuado desarrollo de las actividades de las empresas y el cumplimiento de los objetivos planteados tener una comunicación de punta que permita una interacción eficaz tal como lo menciona (Santana, Cabello, Cubas, \& Medina, 2011), "En las organizaciones, las redes sociales representan los lazos o las relaciones entre los empleados que se forman por el quehacer diario para llevar a cabo trabajos conjuntos, compartir información o conocimiento, ayudarse en la resolución de problemas o, simplemente, relacionarse en actividades extralaborales que pueden ocurrir dentro o fuera del espacio físico laboral".

\section{Reseña histórica}

La tecnología siempre influyó con fuerza en el funcionamiento de las organizaciones a partir de la Revolución Industrial; que fue el resultado de la aplicación de la tecnología de la fuerza motriz del vapor en la producción y que sustituyó inmediatamente el es- 
fuerzo humano, permitiendo el surgimiento de las fábricas e industrias. A finales del siglo XVIII, la invención de la máquina de escribir fue el primer paso para la aceleración del proceso productivo en las oficinas, la invención del teléfono, a finales del siglo XIX, permitió la expansión y la descentralización de las organizaciones rumbo a nuevos y diferentes mercados.

El desarrollo tecnológico siempre constituye la plataforma básica que impulsa el desarrollo de las organizaciones y permite la consolidación de la globalización. Más aún, fue la invención de la computadora en la segunda mitad del siglo XX que permitió que las organizaciones presentaran las actuales características de automatización de sus actividades. Sin la computadora no habría la posibilidad de administrar grandes organizaciones con una variedad increíble de productos, procesos, materiales, clientes, proveedores y personas involucradas. La computadora ofreció a las organizaciones la posibilidad de trabajar con números grandes y con diferentes negocios grandes, simultáneamente, a un costo más bajo y con mayor rapidez y absoluta confiabilidad.

El principio de los años noventa se ve marcado por el surgimiento de la era de la información, gracias al impacto provocado por el desarrollo tecnológico y por la tecnología de la información. La era de la información, implica un inminente y profundo cambio, tanto en el estilo de vida de las personas, como en el ámbito organizacional, el capital financiero cede protagonismo al capital intelectual, "la nueva riqueza es el conocimiento, el recurso organizacional más valioso e importante". (Chiavenato, 2007).

Como resultado del establecimiento de la era de la información, se dio el fenómeno de compactación del espacio físico en las organizaciones, dicha era trajo consigo el concepto de oficina virtual, los antiguos edificios y oficinas fueron drásticamente reducidos de tamaño. La compactación hizo que los archivos electrónicos sustituyeran a los papeles físicos y los muebles donde eran almacenados, traduciéndose en liberación de espacios para otros fines.

Asimismo, surgieron empresas virtuales conectadas electrónicamente, liberando edificios y reduciendo gastos fijos que se hicieron innecesarios. La portabilidad y la virtualidad se transformaron en la nueva dimensión espacial provista por la tecnología de la información.

Las comunicaciones se hacen móviles, flexibles, rápidas, directas y en tiempo real, permitiendo mayor tiempo de dedicación al cliente. La información en tiempo real y en línea (online) permite la integración de varios procesos diferentes en las organizaciones. La información se hace la principal fuente de energía de la organización, su principal y más importante insumo, indispensable para la toma de decisiones.

Tras su creación a principios de los noventas, el Internet, comenzó a influir en la globalización de las empresas y la relación con su entorno (consumidores, proveedores, socios, empleados). Posteriormente sobre el año 1997 con la creación del primer sitio social, la Web 1.0 empezó a evolucionar hacia lo que en 2004 se conoció como Web 2.0.

La Web 2.0 se entiende desde entonces como una infraestructura técnica barata que posibilita los medios colaborativos, interacción y el contenido generado por los usuarios, los cuales son el centro en términos de generación de valor en las empresas. Además, dicha evolución hacia la Web 2.0 transformó el modelo de funcionamiento de las webs pasando de ser plataformas de sólo-lectura a ser muy interactivas con la comunidad y donde se utilizan muchos elementos multimedia (Rodríguez \& Chapis Cabrera, 2019).

\section{Las Redes Sociales}

Aunque actualmente es un tema bastante en boga, su origen se remonta al año 1995

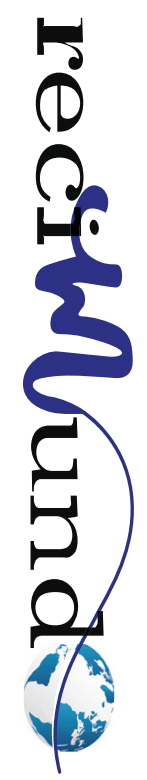


(mucho antes de la aparición de la web 2.0), con la creación del sitio web ClassMates. com, pero no fue hasta 2003 que el término se popularizó con la llegada de sitios web como MySpace y Xing. La popularidad de estos sitios creció rápidamente y grandes compañías tales como Google y Yahoo! en- tran el espacio de las redes sociales en Internet, sin embargo no es hasta la creación de Facebook en 2004 cuando se extiende globalmente el uso de las redes sociales y empieza el crecimiento exponencial en el número de usuarios.

Tabla 1. Diversas definiciones de Red Social

Definiciones de Red Social

Hernández, Posada y Zavala (2013)

- Son estructuras sociales compuestas de grupos de personas, las cuales están conectadas por uno o varios tipos de relaciones, tales como amistad, parentesco, intereses comunes o que comparten conocimientos, e ideas de libre expresión

\section{Santana, Cabello, Cubas y Medina (2011)}

- Son las relaciones interpersonales, no jerárquicas e informales que se forman de manera espontánea dentro de una organización, influyen en el trabajo y la conducta de las personas que la integran y en la actualidad se apoyan en las nuevas tecnologias.

\section{Caldevilla (2010)}

- Son formas de interacción social, definida como un intercambio dinámico entre personas, grupos, e instituciones en contextos de complejidad. Un sistema abierto y en construcción permanente que involucra a conjuntos que se identifican en las mismas necesidades y problemáticas y que se organizan para potencias sus recursos.

Tenzer, Ferro y Palacios (2009)

- Son espacios de encuentro entre individuos, grupos y organizaciones, donde pueden intercambiar contenidos, desarrollar aplicaciones y buscar respuesta a sus inquietudes y necesidades.

Fuente: (Caldevilla Dominguez, 2010)

Aunque aparentemente pueda parecer que el éxito de una red social se mida por su crecimiento rápido a toda la sociedad en general, lo cierto es que han surgido otros tipos de redes más específicas enfocadas hacia un sector concreto del mercado. En cualquier caso, esas plataformas proporcionan, tal como lo exponen diversos autores (reflejado en la Tabla I); la ampliación de relaciones va más allá del contacto físico, permiten instalar en ellas otros tipos de aplicaciones para atraer y retener a los usuarios. No hay datos fiables sobre su grado de aceptación entre los internautas, pero su crecimiento y popularidad ha obligado a muchas compañías y administraciones públicas a restringir su uso dentro de la jornada laboral debido a que la mayoría de redes sociales ofrecen más oportunidades de ocio que aplicacio- nes productivas para la institución.

"Hasta ahora las redes sociales han tenido éxito casi exclusivamente como diversión y entretenimiento, pero bien podrían usarse para realizar trabajos colaborativos entre profesionales y entre organizaciones." (Ros Martin, 2009)

EL crecimiento de usuario de las redes sociales es exponencial y su tendencia a lo largo del siglo XXI ha ido in crecento, de tal forma que los pronósticos muestran que su uso, utilidad y desarrollo seguirá incrementándose con el pasar del tiempo (ver gráfico I); por lo que exige de la administración de las empresas hacer de esta herramienta un instrumento de productividad optimizando su uso y minimizando los riesgos asociados. 
Gráfico 1. Número de usuarios de redes sociales a nivel mundial 2016-2017 y proyecciones 2018-2020

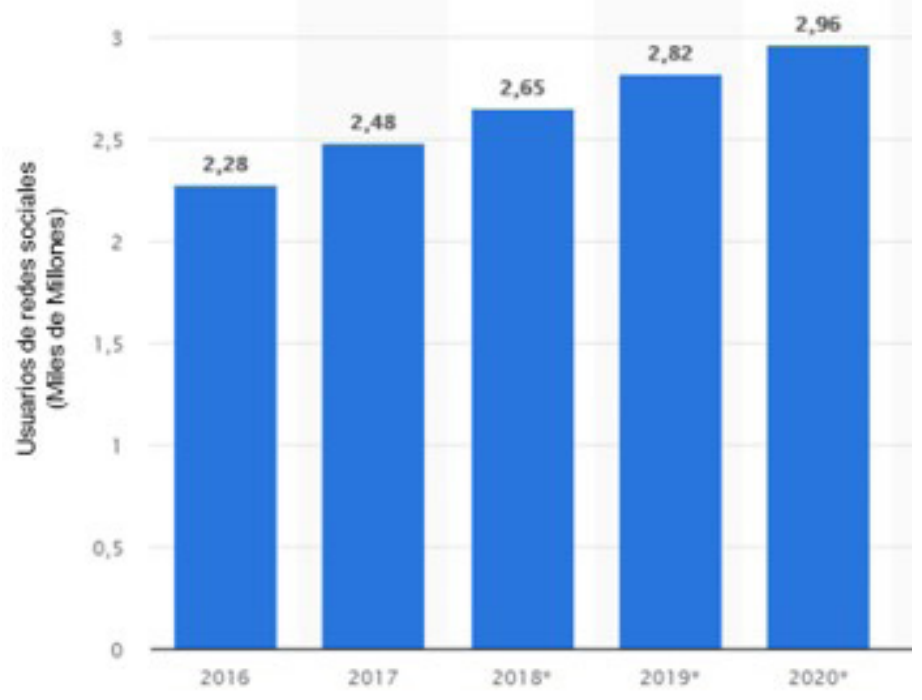

Fuente: (Statista, 2019)

En función de garantizar el uso adecuado y pertinente en la administración, es imporante destacar un de las tipificaciones considerada por (Smith \& McKeen, 2007), que las redes de forma precisa, sus bondades y la diversidad de funciones aplicables a la administración de as empresas su rendimiento. permite dilucidar las funciones y utilidad de

Tabla 2. Clasificación de las redes sociales

Clasificaciỏn de las redes sociales.

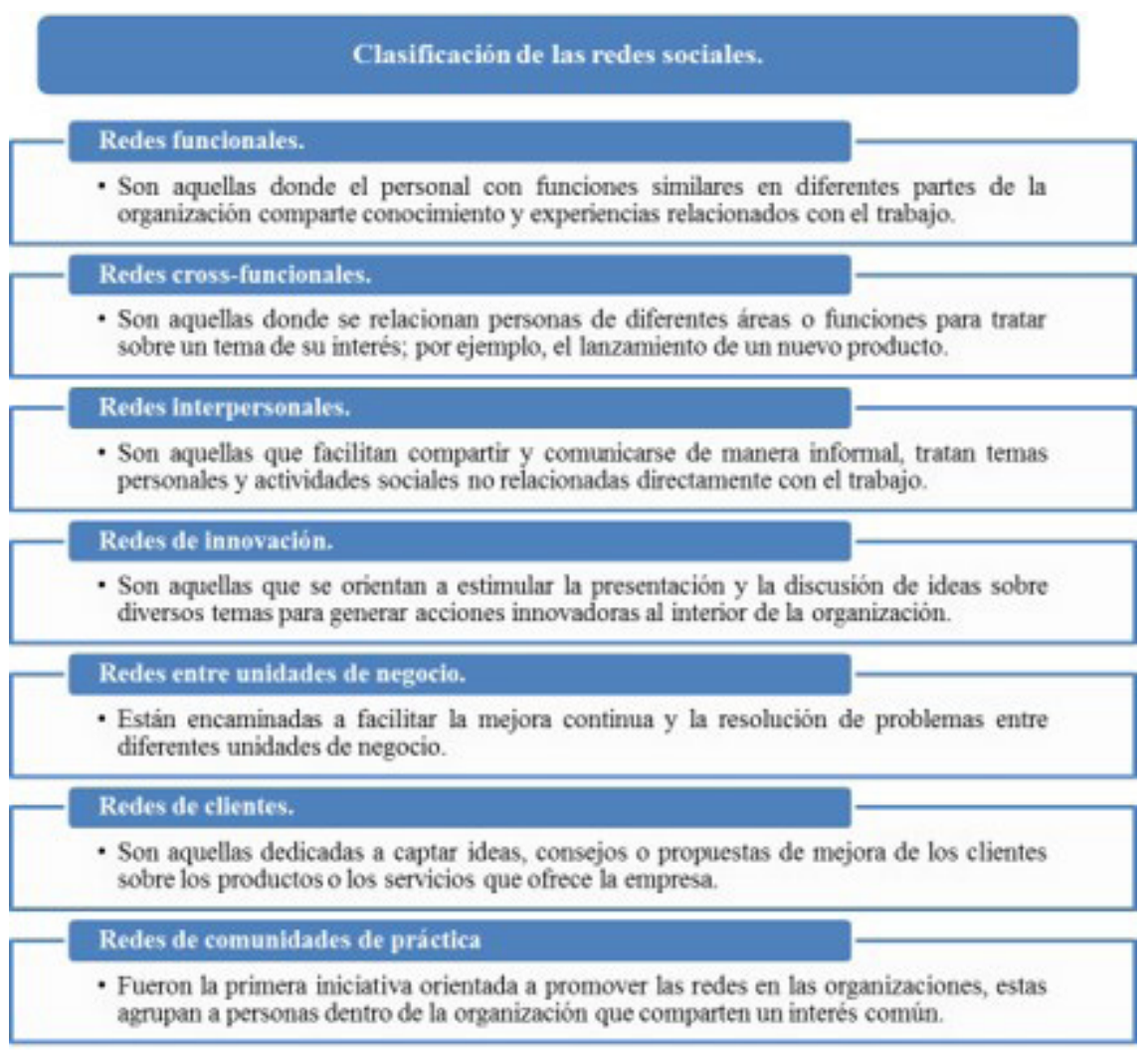

Fuente: (Smith \& McKeen, 2007) citados por (Santana, Cabello, Cubas, \& Medina, 2011) 


\section{Impacto de las Redes sociales en la Ad- ministración de empresa}

En un primer inicio las empresas fueron bastantes resistentes a la idea de implementar esta nueva manera de comunicación corporativa que suponían las redes sociales, se trataba de un nuevo suceso que las organizaciones desconocían, y no tenían idea del alcance que obtendrían.

Según (Savini Somalo, 2014) la incertidumbre de no saber cómo iba a afectar ese hecho al desarrollo de las actividades rutinarias de la oficina, lleva a cabo cierto veto en las distintas páginas web a visitar, dejando prohibida la entrada a páginas como Facebook y Twitter.

Con el pasar de los años, el crecimiento y la popularización de las redes sociales, fueron abriéndole camino en el ámbito corporativo, llamando la atención de la mayoría de las empresas, hasta el punto en el que hoy en día raramente alguna empresa no cuente con al menos un perfil en alguna red social. Se dio paso a nuevos puestos de trabajo exclusivos para el desarrollo de contenido, administración y control de redes sociales en empresas, denominados actualmente con el término de Community Manager.

La radicación de las redes sociales en el ámbito empresarial, supuso un impacto vital en la optimización de la productividad y el incremento de la competitividad en las organizaciones y empresas, siendo impulsado principalmente por el masivo uso que ha dado la sociedad a las nuevas tecnologías de la información y telecomunicaciones.

Este proceso se estructuró en la siguiente forma:

- Inicialmente, se utilizó para para mejorar los procedimientos operativos de orden interno, a través de la introducción de los procesos informáticos en la administración.

- Seguidamente se conectaron los procesos internos de las organizaciones con la sociedad, a través de internet y las nuevas interfaces de comunicación apalancando la mejora de los servicios de forma individual

- Finalmente, para una mayor eficiencia en el proceso comercial de productos y servicios, se aplica la utilización de tecnología con inteligencia artificial, a través de aplicaciones Smart, que permiten la integración de cada uno de los servicios ofrecidos.

En este punto es de vital importancia para las organizaciones, tener en cuenta el impacto que han sufrido con el creciente avance de las tecnologías de información y telecomunicaciones:

- Se modificaron las interrelaciones entre los empleados que interactúan dentro de la misma organización. Todo esto ha hecho necesaria una restructuración en los departamentos de marketing y que las empresas intenten adaptarse a esta nueva tendencia con incertidumbre.

- Provocó una evolución en la forma de relacionarse, interactuar e involucrarse con los consumidores, proveedores y socios, facilitando en la mayoría de los casos la toma oportuna de decisiones, basados en datos reales.

- Se hizo asunto de interés para todas las compañías, suministrar las herramientas necesarias para garantizar la comunicación y el compartir de experiencias entre los consumidores de sus productos y servicios, dado que el control de las comunicaciones ya no recae sobre dichas empresas, razón por la cual, se acentúa la necesidad de recurrir a las redes sociales, como espacios propicios para fomentar tal fin.

Con las nuevas facilidades proporcionadas por las tecnologías de información y telecomunicaciones, la web 2.0 y las redes sociales, las empresas y su administración han logrado la amplificación de sus mercados 
comerciales, obteniendo mejoras significativas en los siguientes aspectos:

1. Intercambio con los consumidores,

2. Intercambio entre los empleados y los proveedores o socios

3. Gestión virtual de los recursos humanos, convirtiéndose en una herramienta de uso común en el reclutamiento del personal

4. Gestión de marketing y comercialización, facilitando el estudio de mercado, la identificación del target y de necesidades inmediatas de los consumidores, entre otras.

Las redes sociales se transformaron en herramientas fundamentales para las empresasen sus procesos administrativos y productivos, abarcando todas las etapas del mismo, amplificando su uso desde el inicio para la captación de potenciales clientes. Con su carácter de globalidad, permite que miles de personas (consumidores) puedan discutir, exponer puntos de vistas, y comentar sus experiencias previa a la compra final del producto o servicio, traduciéndose en un oportunidad perfectamente aprovechable para las organizaciones.

\section{Conclusiones}

- La utilización de las tecnologías de la información dentro de las organizaciones es en una necesidad imperante para las mismas ya que cada día son más accesibles al mercado.

- La estrategia de difusión en redes sociales es una herramienta versátil y eficaz para el posicionamiento de un mercado de las organizaciones debido a que éstas logran el posicionamiento del negocio en la mente del consumidor, para surtir efecto sobre el posicionamiento de una empresa dentro un mercado.

- Estar a la vanguardia en el uso y aprovechamiento de las redes sociales y optimizar los canales de comunicación Cliente/Organización es vital para la lealtad y fidelidelidad del consumidor, ya que a fin de cuentas ya es una realidad en las economías globales.

- Dependiendo de la naturaleza de la empresa, será beneficiosa la limitación del uso de redes sociales dentro del horario laboral, ya que dicha medida impacta directamente sobre la productividad operativa de la misma. En efecto, es responsabilidad de la administración de la empresa trazar políticas regulatorias para maximizar los beneficios y minimizar costos.

Los resultados que se han podido evidenciar, muestran la importancia de la aplicación de las estrategias modernas del marketing de atracción 2.0 en los distintos ambientes de negocios, la utilización de la tecnología genera beneficios en la estrategia utilizada para captar nuevos clientes, posicionarse, generar nombre y poder de marca mediante el buen contenido que las apalanca.

- De la investigación bibliográfica se reconoce que hoy en día son consideradas una herramienta que se han vuelto una necesidad en el desempeño del marketing, iniciaron como una herramienta complementaria de las técnicas tradicionales para la difusión de contenidos y reconocimiento de la marca, convirtiéndose en la actualidad en una necesidad prioritaria a causa de los cambios tecnológicos, a más de que por el simple hecho de no existir estos medios digitales no se podrían aplicar las debidas estrategias.

- Se ha demostrado que son muchos los beneficios del uso de las redes sociales, que su aceptación en los mercados crece de forma acelerada, se reconoce que las redes sociales contribuyen en un porcentaje significativamente en las ventas, deben futuras investigaciones contribuir en cuantificar el impacto de las redes en

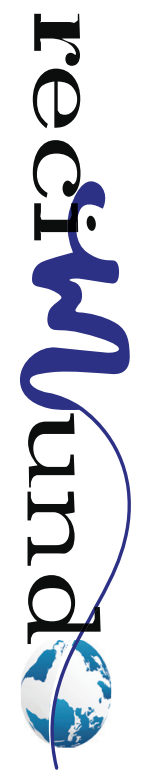


torno a las ganancias de las empresas.

\section{Bibliografía}

Adalpe Nolasco, K. A., Abrego Almazán, D., \& Medina Quintero, J. M. (2016). Análisis de la percepción de uso de las redes sociales como herramienta de marketing en las MiPYMES de Tamaulipas, México. Revista Ibérica de Sistemas y Tecnologías de Información, 18, 49-65.

Caldevilla Dominguez, D. (2010). Las Redes Sociales. Tipología, uso y consumo de las redes 2.0 en la sociedad digital actual. Documentación de las Ciencias de la Información, 33, 45-68.

Chiavenato, I. (2007). Introducción a la teoría general de la administración. Tlalnepantla, Edo. de México: McGRAW-HILL/ÍNTERAMERICANA.

De Castro García, P. M. (2017). IMPACTO DE LAS REDES SOCIALES Y LAS TECNOLOGÍAS SMART SOBRE LA ACTIVIDAD ECONÓMICA Y EMPRESARIAL. Santander.

Ellison, N., Steinfield, C., \& Lampe, C. (2007). The benefits of Facebook "friends":social capital and college students. Journal of computermediated communication, 12(4), 1143-1168.

Hernandez, M., Posada, K., \& Zavala, Y. (2013). Influencia del facebook en el desempeño académico de alumnos/as de tercer ciclo del centro escolar Santiago Orellana Zelaya de la ciudad de concepción Batres, departamento de Usulután, durante el año 2013. El Salvador.

Rodríguez, V. A., \& Chapis Cabrera, E. (2019). Importancia de las tecnologías de la información y las comunicaciones, el internet y las redes socia- les en el mejoramiento y desarrollo de las empresas. Contribuciones a la economía, 1-10.

Ros Martin, M. (2009). Evolución de los servicios de redes sociales en internet. El profesional de la información, 18(5), 552-557.

Salas Narváez, L. C., Acosta Véliz, M. M., \& Jiménez Cercado, M. E. (2018). Importancia del Marketing de Atracción 2.0, en las Pequeñas y Medianas Empresas de la ciudad de Guayaquil (Ecuador). Revista Espacios, 39(18), 31.

Santana, M., Cabello, J., Cubas, R., \& Medina, V. (2011). Redes sociales como soporte a la gestión del conocimiento. Lima: Esan Ediciones.

Savini Somalo, R. S. (2014). Impacto de las redes sociales en la empresa. España: Universidad de la Rioja - Servicio de Publicaciones.

Smith, H. A., \& McKeen, J. D. (2007). Developments in practice XXVI. Social Networks: knowledge management's "killer app"? Communications of the Association for Information Systems, 18, 611-621.

Statista. (19 de 06 de 2019). Statista - Número de usuarios de redes sociales a nivel mundial entre 2016 y 2021. Recuperado el 02 de 2020, de https://es.statista.com/estadisticas/512920/numero-mundial-usuarios-redes-sociales/

Tenzer, S. M., Ferro, O., \& Palacios, N. (2009). StudyLib. Recuperado el 02 de 2020, de https://studylib. es/doc/7013816/redes-sociales-virtuales--personas--sociedad-y-empresa-co...

Yaguana Venegas, L. A. (2018). Redes sociales: herramienta o necesidad en el desempeño del marketing en la ciudad de Machala. Machala.

\section{CITAR ESTE ARTICULO:}

Hugo Cardenas , F., Jimenez Rosero, C., Holovatyi, M., \& Lara Pazos, P. (2020).

El impacto de las redes sociales en la administración de las empresas. REClMUNDO, 4(1), 172-181. doi:10.26820/recimundo/4.(1).enero.2020.172-181 